Questions vives

\section{Questions Vives}

Recherches en éducation

$\mathbf{N}^{\circ} 33 \mid 2020$

Visées heuristiques, praxéologiques et critiques dans les recherches participatives en éducation

\title{
Les impasses critiques de la recherche participative : leçons tirées de débats épistémologiques en sociologie critique
}

Participatory Action Research Deadlocks: Lessons from Critical Sociology

\section{Arianne Robichaud et Marina Schwimmer}

\section{(2) OpenEdition}

Édition électronique

URL : http://journals.openedition.org/questionsvives/4713

DOI : 10.4000/questionsvives. 4713

ISSN : 1775-433X

Éditeur

Université Aix-Marseille (AMU)

Édition imprimée

Date de publication : 15 décembre 2020

ISBN : 978-2-912643-57-5

ISSN : 1635-4079

Référence électronique

Arianne Robichaud et Marina Schwimmer, «Les impasses critiques de la recherche participative: leçons tirées de débats épistémologiques en sociologie critique », Questions Vives [En ligne], № 33 | 2020, mis en ligne le, consulté le 25 mars 2021. URL : http://journals.openedition.org/questionsvives/ 4713 ; DOI : https://doi.org/10.4000/questionsvives.4713

Ce document a été généré automatiquement le 25 mars 2021.

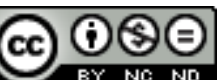

Questions Vives est mis à disposition selon les termes de la licence Creative Commons Attribution Pas d'Utilisation Commerciale - Pas de Modification 4.0 International. 


\title{
Les impasses critiques de la recherche participative : leçons tirées de débats épistémologiques en sociologie critique
}

\author{
Participatory Action Research Deadlocks: Lessons from Critical Sociology
}

Arianne Robichaud et Marina Schwimmer

\section{Introduction}

1 La nature critique de la recherche participative (RP) est généralement admise d'emblée : en déstabilisant et en reformulant les rapports habituels aux savoirs et les hiérarchies entre experts et acteurs du terrain, l'épistémologie des principales déclinaisons de la RP (recherche collaborative, recherche-action, recherchedéveloppement, recherche-formation) fracture les conceptions traditionnelles du chercheur et de l'acteur/praticien, voire du citoyen. En ce sens, elle permettrait le développement du pouvoir d'action du citoyen, la valorisation du savoir du praticien et favoriserait une prise de conscience des capacités des sujets (Anadón, 2007, p.15) maintenant mieux outillés, par l'expérience d'une RP, à agir sur les situations quotidiennes qui freinent ou limitent leur potentiel d'émancipation individuelle ou collective. Le langage et le vocabulaire paraissent ainsi éminemment critiques: aplanissement des hiérarchies épistémologiques, pouvoir d'action de l'acteur, prise de conscience émancipatoire. Toutefois, et tel est l'objectif du présent texte, les fondements de la RP ne peuvent se soustraire à une réflexion substantielle sur ses liens épistémologiques à la notion même de critique, et sur leur récupération par un système d'organisation de la recherche qui, malgré l'effort des projets participatifs visant à lui résister par les moyens d'une démocratisation du savoir, contribue possiblement à idéologiser la RP. 
Cet article propose une remise en question des « gestes critiques » de la RP en montrant leurs limites théoriques et pratiques. Après une courte présentation des liens entre la notion de critique en sciences sociales et les prémisses critiques de la RP, trois «impasses critiques" sont présentées: 1) les possibilités d'instrumentalisation des participants en RP, 2) les difficultés organisationnelles et symboliques à réellement disloquer les rapports de force entre chercheurs et participants dans ce type de recherche, puis 3) la façon dont ses assises et méthodes sont sujettes à une récupération idéologique par différentes instances influentes et décisionnelles dans le monde de la recherche. Chacune de ces impasses de la RP est analysée à la lumière du débat épistémologique entourant depuis quelques décennies la sociologie critique, qui oppose en France les défenseurs d'une sociologie de la domination (bourdieusienne) à ceux d'une sociologie de la critique (boltanskienne), puis ceux des différentes générations de l'École de Francfort (Horkheimer et Adorno «face» à Habermas et Honneth) en Allemagne. L'argumentaire traversant l'ensemble du texte, possiblement à contrecourant de ce qui est présentement de mode en sciences humaines et sociales, cherche à restituer la valeur des "premières leçons » de la sociologie critique (Horkheimer, Adorno, Bourdieu) pour penser le potentiel des gestes critiques de la RP ; leçons qui, si elles sont présentes (dans une mesure relative) au sein des RP, nous semblent toutefois être reléguées au second plan derrière les postulats d'une sociologie critique de «l'acteur ». Au final, cet article souhaite donc contribuer à une discussion de fond sur la dimension critique de la RP, en la soumettant et la confrontant aux traditions sociologiques ayant fait de la critique l'un de leurs principaux objets, sinon leur principal intérêt.

\section{La critique en recherche participative et la critique en sciences sociales : confrontation à la sociologie critique et à ses débats épistémologiques}

Circonscrire ce qu'est la critique, son état et ses visées, n'est pas chose aisée. Au sein même des disciplines philosophiques et sociologiques l'invoquant, la critique est souvent décrite comme étant "en crise» (Trom, 2008, p.108), engluée dans les différentes traditions théoriques qui l'ont faite émerger, ou encore reprise, réappropriée et "désarmée » (Boltanski et Chiapello, 1999) par les pouvoirs en place, neutralisant par le fait même sa raison d'être ou son impulsion première de remise en question des institutions et rapports de force dominants. En termes épistémologiques, la sociologie critique serait prise entre Bourdieu et Boltanski en France ou entre la première et les deuxième et troisième générations de l'École de Francfort en Allemagne (Fassin, 2009, p. 199), c'est-à-dire entre une sociologie de la domination et une sociologie chargée de mettre en lumière le potentiel critique et réflexif des acteurs sociaux. Autrement dit, la critique de la domination (qu'elle s'inscrive en territoire français ou allemand), qui se donne généralement pour tâche de dévoiler les rapports de force et les idéologies dominantes contenues et disséminées par les institutions puis de caractériser l'aliénation politique, économique et culturelle du sujet moderne, est bousculée et remise en question par les impératifs d'une prise en compte des capacités critiques et communicationnelles de l'acteur : dépendante d'un théoricien surplombant observant la réalité du haut de sa spécialisation et de ses privilèges, elle ne serait plus à 
même de décrire avec acuité les faits sociaux réels et, surtout, de rendre une voix au sujet « ordinaire » conçu comme acteur critique.

Les arguments généraux sont connus : la logique de la domination ne caractérise pas l'ensemble de la vie sociale, les agents ne sont pas nécessairement aveuglés par leurs propres illusions, et la connaissance critique du chercheur n'est pas nécessairement supérieure à celle de l'acteur, nous dit ainsi Boltanski (2009) ; même « rupture » dans la théorie critique allemande, qui opposerait les premiers penseurs de l'École de Francfort (Horkheimer, Adorno) aux plus récentes générations représentées par Habermas et Honneth, ces derniers ayant réussi (grâce au tournant linguistique habermassien ou au concept honnethien de reconnaissance) à ébranler les conceptualisations surplombantes de leurs prédécesseurs en offrant des théorisations qui reconnaissent la capacité d'action et d'émancipation des individus (Frère, 2015). Nous parlons donc, en ce sens et pour les besoins précis de l'exercice ici proposé, d'une sociologie critique de la domination et d'une sociologie critique de l'acteur et de la communication dans une optique qui n'est pas celle de la chronologie historique : plutôt, à gros traits, dans le sens d'une première sociologie critique plus déterministe qui s'intéresse à la domination, à l'aliénation et à la reproduction dans une perspective verticale, et d'une deuxième sociologie critique qui tente de mettre en lumière l'horizontal des faits sociaux (intersubjectivité communicationnelle entre acteurs, reconnaissance, agentivité critique de l'acteur social), et ce, sans nécessairement nier les enchevêtrements entre l'une et l'autre.

Cette «double » prémisse est habituellement présente, à différents degrés, dans la perspective épistémologique générale de la RP, même si l'histoire de son développement ne montre pas un attachement spécifique à la sociologie critique : en effet, les références théoriques initiales de la RP seraient plutôt ancrées dans les travaux de John Dewey, de Kurt Lewin, de Paulo Freire ou de Donald Schön (Bourrassa et al., 2007, p. 3). De plus, la RP ne recouvre pas un ensemble unique de principes, de postures et de méthodologies, chacune de ses variantes s'inscrivant dans des domaines variés (éducation, travail social, santé, pratiques communautaires, etc.), mais également dans des traditions théoriques et culturelles différentes. Il n'en demeure pas moins que la RP et ses multiples déclinaisons partagent certains postulats fondamentaux avec les deux formes de sociologie critique décrites ci-dessus, notamment les visées émancipatoires d'une activité sociale transformée: la RP est attachée aux idées d'engagement social et d'action culturelle, cherche à lier action et savoir, et s'inscrit dans un mouvement de conscientisation et d'émancipation " visant à produire une réflexion critique engagée dans un processus de recherche et de changement social » (Bourrassa et al., 2007, pp. 5-7). Par contre, il est clair que la RP privilégie ultimement une perspective sociologique "horizontale»: en effet, si la science et la façon dont elle est institutionnalisée et reconduite sont reconnues au sein des RP comme étant traditionnellement positivistes et élitistes sur le plan de la validité des savoirs et des individus reconnus dans ses activités (ce qui constitue un écho aux différentes thèses de Horkheimer et de Adorno $(1937$; 1947) sur l'hégémonie idéologique de la pensée scientifique dans la société moderne, ou encore à celles de Bourdieu (1987) sur la domination symbolique exercée par l'expert), la RP considère généralement qu'elle peut contribuer à une fracture des hiérarchies classiques de la recherche (chercheur/praticien, savoir scientifique/sens commun, etc.) par la reconnaissance du savoir (critique) de l'acteur. Ainsi, à l'image du postulat boltanskien 
suivant lequel « les jugements moraux que les acteurs formulent dans le cours de leurs activités quotidiennes prennent souvent la forme de critiques » (Boltanski, 2009, p. 19), ou encore de l'idée habermassienne selon laquelle l'agir communicationnel des acteurs sociaux peut toujours représenter un potentiel de résistance face à une rationalisation instrumentale des modes de vie (Habermas, 1987, p. 401), la RP fait souvent, au final, le même pari que celui de la sociologie critique de l'acteur et de la communication.

Ce sont les apories de ce pari que nous tentons de relever dans les pages qui suivent, à l'image de certaines critiques ayant été adressées à Boltanski et Habermas au cours des dernières décennies et suivant différentes limites des RP relevées dans la littérature scientifique. À partir du moment où nous reconnaissons que les RP s'effectuent rarement en marge des rouages administratifs et organisationnels de l'université, d'organismes gouvernementaux, d'institutions étatiques ou d'entreprises, dans quelle mesure peut réellement s'actualiser, dans la communication entre le chercheur et les acteurs concernés, le potentiel critique des participants? Ce n'est donc pas l'idéal d'émancipation des acteurs, ou encore celui de leur implication directe dans les RP qui sont, ici, mis en procès : nous nous attardons plutôt aux mécanismes et aux processus par lesquels la RP, du fait qu'elle est liée à différentes contraintes financières, organisationnelles, institutionnelles, est susceptible de se retourner contre elle-même. Dès lors, quelles sont les avenues critiques possibles pour la RP ?

\section{Les impasses critiques de la RP}

7 Quelques études, outre les RP spécifiques qui font état des limites de leurs entreprises particulières, s'attardent spécifiquement aux difficultés de la RP à rencontrer ses propres objectifs de dé-hiérarchisation scientifique et épistémologique. Appuyée sur une recension interdisciplinaire de la littérature (23 champs disciplinaires, plus de 70 études recensées) portant sur des pratiques de RP menées partout dans le monde dans les 20 dernières années, Lake et Wendland (2018) mettent en lumière trois dérives potentielles de la RP : 1) l'instrumentalisation des participants (et non l'intégration et l'implication réelles de ces derniers) (p. 21), 2) le maintien des rapports de force entre chercheurs et participants (p. 23), puis 3) la régulation externe des communautés (p. 23) dissimulée sous un vocabulaire progressiste, participatif et communautaire, signe d'une possible récupération idéologique des assises et méthodes de la RP. Ces manifestations ne sont pas étrangères aux analyses respectives des sociologues de la domination comme de ceux de l'acteur ou de la communication, qui nous offrent à la fois des avenues complémentaires et des perspectives concurrentes pour penser les limites critiques ci-haut mentionnées de la RP.

\subsection{Les possibilités d'instrumentalisation des participants en RP}

8 D'abord, l'instrumentalisation des participants, aux dépens de leur intégration et implication réelles, pose le problème de l'autonomie et de la reconnaissance de l'acteur social dans une situation qui, pourtant, a spécialement été conçue en référence à des objectifs d'autonomie et de reconnaissance. Dans cette perspective, le participant n'est pas un sujet à part entière qui fait la recherche : il est un sujet qui permet quelque chose d'autre, qui permet l'atteinte d'un objectif qui, aussi noble soit-il, le transforme au passage en matériau, en informateur (Lake et Wendland, 2018, p. 21), en instrument 
visant l'élucidation d'une question qui, si elle le concerne intimement, est ultimement traitée en extériorité. La complexité de l'enjeu est donc particulièrement profonde : comment des recherches souhaitant spécifiquement reconnaitre et permettre la voix des acteurs sociaux peuvent-elles se retourner, malgré elles, contre ce même principe ? Ce risque d'instrumentalisation est, pourtant, bien connu et considéré par les chercheurs œuvrant dans le cadre de RP :

Rappelons que l'étiquette « participatif » ne présume en rien du niveau de participation recherché et réel, et on ne peut nier que le risque d'instrumentalisation de la participation dans le processus existe. $\mathrm{Si}$, dans certains cas, les participants deviennent eux-mêmes des cochercheurs, dans d'autres études, ils vont simplement légitimer la démarche. (René et al., 2013, p. 8)

Différents éléments sont alors à considérer, qu'ils s'inscrivent du côté du chercheur, du participant, de la méthodologie préconisée ou de la logique institutionnelle dans laquelle s'insère la RP : le chercheur a-t-il posé les conditions nécessaires permettant une résistance à cette instrumentalisation ? Le participant s'est-il lui-même engagé de façon suffisante dans la démarche pour prévenir cette (auto)-instrumentalisation ? La méthodologie choisie reconduit-elle, consciemment ou non, une conception traditionnelle du participant conçu comme "source d'informations» et non comme cochercheur à part entière? La logique institutionnelle dans laquelle s'inscrit une RP donnée (canevas validé par l'université ou l'organisme subventionnaire) oblige-t-elle implicitement une telle instrumentalisation? En opposition à Bourdieu, Boltanski (1990) nous invite à laisser de côté les hypothèses suivant lesquelles le chercheur, du haut de son statut social privilégié (et du fait qu'il représente notamment l'institution universitaire et puisse être bénéficiaire de fonds gérés par des instances décisionnelles), reconduirait une forme de domination à l'égard du participant, ou que ce dernier contribuerait lui-même, de façon plus ou moins consciente, à son aliénation, à son exclusion symbolique et à sa propre instrumentalisation :

11 Les acteurs que ces travaux [les travaux issus de la sociologie pragmatique de la critique] ont donné à voir étaient très différents des agents figurant dans la sociologie critique de la domination. Ils étaient toujours actifs, et non passifs. Ils étaient carrément critiques, et même critique un peu à la façon des sociologues critiques, ne cessant de dévoiler leurs intentions et les travers cachés de leurs adversaires - souvent rapportés à leur position sociale -, en mobilisant, à cet effet, des schèmes empruntés à la sociologie critique, diffusés par l'enseignement ou par les médias. Ils faisaient valoir leurs revendications, dénonçaient des injustices, produisaient des preuves à l'appui de leurs plaintes ou forgeaient des arguments pour se justifier face aux critiques dont ils faisaient euxmêmes l'objet. Envisagé de ce point de vue, le monde social n'apparaît pas comme le lieu d'une domination subie passivement et inconsciemment, mais plutôt comme un espace traversé par une multitude de disputes, de critiques, de désaccords et de tentatives pour réinstaurer localement des accords toujours fragiles. (Boltanski, 2009, p. 51)

12 Toutefois, quelques constats issus de différentes RP (Musil, 2018; René et al., 2009; Hagey, 1997aHh) nous obligent à ne pas balayer si rapidement du revers de la main les thèses bourdieusiennes. On note par exemple un empowerment limité par le manque d'intérêt des participants face à une situation qu'ils interprètent comme étant impossible à transformer, phénomène que nous pourrions attacher au concept bourdieusien de champ (Bourdieu, 1984) : l'idée n'est pas que l'acteur soit complètement 
aliéné et incapable de reconnaître une injustice ou une situation qui le désavantagent, mais que sa position et sa marge de pouvoir dans un contexte donné soient tellement limitées par un rapport de force à l'avantage des dominants que l'action apparaît inutile, vaine. Comme le souligne Bourdieu,

La lutte présuppose un accord entre les protagonistes sur ce qui mérite qu'on lutte et qui est refoulé dans le cela-va-de-soi, laissé à l'état de doxa, c'est-à-dire tout ce qui fait le champ lui-même, le jeu, les enjeux, tous les présupposés qu'on accepte tacitement, sans même le savoir, par le fait d'entrer dans le jeu. (1984, p. 115)

En ce sens, "même les acteurs dans une position subordonnée et engagés dans des luttes de subversion contribueraient donc à la reproduction du champ plutôt qu'à son renversement " (Ancelovici, 2009, p. 46), à l'image d'une RP qui, même si elle tente de donner une voix aux acteurs, est peut-être incapable de suffisamment bouleverser les rapports de forces à l'intérieur du champ dans laquelle elle se déroule pour engager les acteurs. Suivant une logique bourdieusienne, il est possible que ces derniers soient ainsi condamnés au statut de simples instruments méthodologiques plutôt qu'à celui d'agents actifs, et ce, malgré les intentions initiales des chercheurs et des participants eux-mêmes.

Dans la perspective de l'École de Francfort, la question de l'instrumentalisation des individus évolue au fil de ses générations successives. Avec Horkheimer et Adorno (1947), elle est d'abord la conséquence de l'hégémonie, dans les sociétés capitalistes avancées, de la rationalité instrumentale comme logique d'organisation sociale : «pour les gouvernants, les hommes deviennent du matériel, comme la nature toute entière devient du matériel pour la société» (p. 135). L'individu est donc objectivé, instrumentalisé pour servir autre chose que sa propre émancipation, dans une perspective néo-marxiste assez classique (accumulation du capital et du pouvoir par les dirigeants, domination sociale par l'idéologie de consommation de masse, etc.). Cette thèse nous obligerait à concevoir, pour le phénomène d'instrumentalisation des participants en RP, le chercheur comme un "gouvernant ", comme un "puissant », ce qui nous semble peu réaliste; toutefois, la thèse de la rationalité instrumentale comme logique d'organisation sociale n'est peut-être pas à écarter pour autant. Avec Habermas, le processus de rationalisation instrumentale se complexifie : $s$ 'il reproche à Horkheimer et Adorno d'absolutiser le concept de rationalité instrumentale aux dépens d'une rationalité communicationnelle qui vise l'intercompréhension des acteurs, il reconnaît tout de même que la pression exercée par la logique instrumentale des systèmes et sous-systèmes modernes (État, économie, bureaucratie, administration, juridique) parvient, dans plusieurs cas, à coloniser le monde vécu des acteurs, et donc les mécanismes langagiers qu'ils utilisent dans leurs vies quotidiennes :

16 Aujourd'hui, par le moyen de l'argent, de l'organisation et de la décision administrative, bien d'autres domaines d'action se sont de la même façon autonomisés en systèmes; ils phagocytent des modes de vie et les dégagent de formes d'intégration sociale d'apparence démodée et coûteuse - une intégration qui passait par les valeurs, par les normes et par un accord obtenu dans la communication. (...) Cette rupture doit et peut favoriser l'émancipation de rapports de domination figés; mais elle entraîne d'un autre côté le risque d'un tarissement bureaucratique des formes de communication, c'est à-dire le danger d'une formalisation qui ne sera nullement libératrice mais plutôt porteuse de mort puisqu'elle frappera des relations qui, par essence, ne sont pas formalisables. (...) En se propageant à des domaines d'existence qui 
obéissaient aux lois propres d'une rationalité pratique d'ordre moral et esthétique, les formes de la rationalité économique et de la rationalité administrative conduisent à une sorte de «colonisation» du monde vécu. J'entends par là l'appauvrissement des possibilités d'expression et de communication, lesquelles, pour autant que nous puissions en juger, restent encore nécessaires dans des sociétés complexes pour que les individus puissent apprendre à se trouver eux-mêmes, à maitriser leurs conflits propres et à régler en commun, des conflits collectifs. (Habermas, 1979, p. 44)

Dans cette perspective, l'instrumentalisation des participants en RP serait le signe d'un appauvrissement de la communication intersubjective entre chercheurs et participants, dû à l'intrusion d'une logique organisationnelle/formelle dans le processus de recherche. Ce genre de phénomènes est notamment soulevé par BrydonMiller (2013) et Giles (2012) qui mentionnent les difficultés des RP à rencontrer leurs objectifs d'implication réelle et entière des participants face aux impératifs de fonctionnement de la recherche liés à l'octroi de subventions, à l'accès au terrain, au temps et aux ressources allouées, soit des problèmes liés à une infrastructure de recherche dont la logique (efficacité, atteinte d'objectifs circonscrits, délais précis) diffère fondamentalement d'un exercice d'intercompréhension, de partage langagier et d'influences mutuelles entre chercheurs et participants. En ce sens, la dimension critique des RP fait tout de même le pari d'une perspective (habermassienne) qui reconnaît le potentiel de résistance de l'agir communicationnel entre acteurs face aux logiques instrumentales du système de la recherche universitaire, résistance qui constitue même explicitement, dans plusieurs RP, l'un de ses objectifs précis. Toutefois, il faut aussi reconnaître la force de la logique administrative dans laquelle s'inscrit les $\mathrm{RP}$, comme l'illustrent les phénomènes d'instrumentalisation de ses participants : dit simplement, une RP soucieuse de rencontrer complètement ses objectifs d'égalité entre chercheurs et participants est-elle même possible au sein d'un système de recherche universitaire, gouvernemental ou entrepreneurial ? La réponse à cette question touche directement les nuances entre la théorie critique de Horkheimer/Adorno et celle de Habermas : la RP peut être considérée comme une pratique qui, ultimement, est ravalée par le fonctionnement totalisant de la société administrée, ou comme une entaille susceptible de déstabiliser et d'agir comme une contre-force à cette société administrée.

\subsection{Les difficultés organisationnelles et symboliques à disloquer les rapports de force entre chercheurs et participants en RP}

18 Ensuite, les difficultés organisationnelles et symboliques à réellement disloquer la relation traditionnelle entre chercheurs et participants posent le problème classique des rapports de force entre membres d'une classe "spécialisée » et membres d'une classe "non-spécialisée » dans le contexte précis d'une recherche institutionnalisée. Ici aussi, donc, phénomène contradictoire : la RP, malgré ses prémisses d'aplanissement de la hiérarchie entre le chercheur et le participant, ne parviendrait pas nécessairement à renverser ce rapport. Nous pouvons entre autres invoquer, pour expliquer le problème, le concept bourdieusien de domination symbolique appliqué au rapport entre un expert et un praticien/citoyen dans le cadre d'une recherche : « le privilège qui est au principe de toute activité théorique, en tant qu'elle suppose une coupure épistémologique, mais aussi sociale, ne gouverne jamais aussi subtilement cette activité que lorsque, faute de s'apparaître comme tel, il conduit à une théorie implicite de la pratique qui est 
corrélative de l'oubli des conditions sociales de possibilité de la théorie » (Bourdieu, 1972, p. 158). Autrement dit, les privilèges symboliques d'une classe sociale donnée, malgré les tentatives d'aplanissement dans son rapport à autrui, rattrapent et caractérisent toujours l'activité scientifique menée dans le cadre d'institutions fondamentalement "gardiennes » de ces privilèges (universités, centres de recherche, organismes subventionnaires qui permettent la conduite de ces recherches). Mais Boltanski s'oppose :

Les problèmes que nous as posés la façon dont la notion de domination était mise en œuvre dans la sociologie critique dérivent de son caractère à la fois puissant et trop vague. L'usage extensif de la notion de domination conduit à envisager à peu près toutes les relations entre acteurs dans leur dimension verticale, depuis les relations hiérarchiques explicites, jusqu'aux liens les plus personnels. Du même coup, ce que le sociologue considèrera, dans une optique critique, comme étant une relation de domination n'est pas nécessairement présenté, ni encore moins vécu, par les acteurs dans ce registre, et ces derniers peuvent même se montrer offensés par une telle description. (Boltanski, 2009, pp. 41-42)

Cette idée rejoint les principes d'horizontalité de certaines recherches s'inscrivant dans le courant de la RP, suivant lesquels le savoir de l'ensemble des participants à la recherche est reconnu: par rebond, le pouvoir y est davantage partagé, redistribué de façon plus équitable entre les acteurs. Comme le souligne Morrissette (2013) à propos de la recherche-action, « elle se distingue d'autres types de recherches par son mode de cogestion : chercheurs et acteurs sociaux sont partenaires dans toutes les étapes de la recherche» (p. 37). Il s'agit notamment de sortir la recherche des laboratoires en s'intéressant, avec les groupes concernés, à des problèmes concrets mais surtout, depuis quelques décennies, à concevoir l'acteur (le praticien, le citoyen, le professionnel) comme un agent réflexif qui « réfléchit sur et dans l'action» (ibid., p. 38). Cela procède, surtout dans les RP francophones, d'un emprunt théorique à la critique de l'épistémologie positiviste de Donald Schön (1983) nous permettant de voir comment l'engagement de praticiens réflexifs est "essentiel dans l'amélioration de leurs pratiques, ceux-ci étant capables de se donner les moyens de contrôler leur action, de remettre en question leurs postulats, d'examiner leurs pratiques » (ibid., p. 38).

21 Il se peut toutefois que la reconnaissance, tout à fait légitime, du potentiel critique des acteurs se soit opérée dans le cadre d'un mouvement qui, paradoxalement, tend à neutraliser et diluer le potentiel critique du chercheur : la peur d'adopter une posture surplombante, de reconduire une conception élitiste et hiérarchisée de la recherche et de ne pas laisser l'avant-scène aux discours des participants semble avoir vidé, dans plusieurs RP, la possibilité de contribution du chercheur qui devient un facilitateur plutôt qu'un acteur critique à part entière dans la recherche. Ces différentes précautions paraissent compréhensibles dans l'optique d'un "retour du balancier», qui exige souvent qu'une posture radicale soit empruntée pour réellement transformer un état de fait inégalitaire, comme celui d'une science sociale plus traditionnellement préoccupée à prendre l'acteur comme objet d'analyse que comme partenaire. Mais il se peut aussi que la RP adopte une conception du dialogue et du partenariat qui soit davantage politically correct que réellement transgressive, et ce, d'une façon qui, au final, va à l'encontre de l'objectif d'une reconnaissance réelle des capacités critiques des acteurs ; et certaines RP semblent également pousser la facilitation à l'extrême, en offrant non pas une conception de l'acteur capable de critique, mais bien la conception 
infantilisante d'un acteur qui serait confus devant le vocabulaire "théorique » du chercheur :

La recherche-action est aussi le lieu d'une véritable remise en question de la division sociale du savoir et du pouvoir entre les différents partenaires impliqués. Au-delà des différences propres des savoirs pratiques et du savoir théorique, il existe souvent des écarts culturels importants entre le chercheur, le praticien professionnel et les clientèles impliquées qui proviennent de catégorie ou de classes sociales différentes. Il devient difficile d'expliquer le concept d'aliénation à un groupe d'ouvriers manuels et l'explication risque de viser tout aussi bien la culture savante! (Rhéaume, 1982, p. 50)

Est-ce que le fait d'être un ouvrier implique l'incapacité de tenir une discussion de fond sur les rapports entre son corps de travail et le patronat, sur ses conditions de travail spécifiques, sur les liens que l'ouvrier entretient avec l'objet de son travail? Au contraire, et la grande majorité des RP le reconnaissent: le praticien, le citoyen, le travailleur est dans la meilleure position pour témoigner des tenants et aboutissants de son activité. Mais il nous semble que la peur du théoricien surplombant, légitime dans certains aspects de la RP, ne doive pas non plus mener à une négation du potentiel critique qu'une discussion "confrontante » entre les savoirs du chercheur et ceux du participant peut contenir. Ici, confrontation ne signifie pas domination, mais plutôt reconnaissance: elle signifie que, par un exposé de certains concepts propres à la sociologie critique, le chercheur reconnaît les facultés critiques du participant en lui permettant de remettre en question ces concepts, et non en les lui «cachant » sous prétexte qu'il ne serait pas en mesure de les comprendre. Un retour à Horkheimer permet de bien illustrer le fonctionnement de cette dialectique de la pensée critique :

Le déroulement du débat entre les éléments les plus avancés du prolétariat et les individus qui énoncent la vérité à son sujet, ainsi qu'entre ces éléments avancés et leurs théoriciens d'une part, et d'autre part le reste du prolétariat, doit être compris comme un processus d'influence réciproque dans lequel la conscience se développe, en même temps que ses énergies libératrices, ses énergies motrices, agressives, et son action disciplinante. L'acuité de ce débat se manifeste dans la possibilité toujours présente d'une tension entre le théoricien et la classe à laquelle s'adresse sa pensée. (Horkheimer, 1937, pp. 48-49)

Cette perspective n'est, généralement, pas celle des RP, où l'accent est davantage posé sur la co-construction, la collaboration, l'effacement relatif du chercheur et la mise en lumière du savoir de l'acteur: habituellement, la RP n'use pas d'un vocabulaire conflictuel, antagoniste. Pourtant, elle pourrait y gagner sur le plan de la critique, comme le soulignent les quelques auteurs s'intéressant à radicaliser le geste critique des RP : « ces recherches ne doivent pas forcement aboutir à une méthode ou un savoir commun. Le fait de connaitre les différences (antagonismes) serait un point de départ pour construire par exemple une démocratie radicale ou agoniste » (Granosik, 2018, p. 79), au sens où l'entend Mouffe (2000). Et au sein des débats qui nous intéressent ici, il s'agit d'une forme de contre-argument à la pensée délibérative habermassienne qui, privilégiant l'atteinte d'un consensus intersubjectif, passe peut-être à côté d'une des sources fondamentales de la critique : l'exercice dialectique de confrontation qui ne mène pas nécessairement à un accord, mais à des positions différenciées desquelles peuvent naître de nouvelles formes de critiques. 


\subsection{La récupération idéologique des assises et des méthodes de la RP par différentes instances influentes et décisionnelles dans le monde de la recherche}

nalement, la RP se trouve également au cœur d'enjeux de récupération idéologique par les instances décisionnelles chargées de la financer, ou encore par les entreprises ou associations qui adoptent ses assises et ses méthodes: comme le précise Anadón (2007, p. 16), «les finalités de la recherche participative peuvent passer d'une orientation critique qui promeut un changement radical à une orientation plus technique qui cherche un changement mieux "adapté" au fonctionnement des organisations existantes ». On peut ainsi se demander, avec Jordan (2013), si le fait que la recherche participative soit de plus en plus adoptée par des organisations privées ne témoigne pas du fait que cette dernière soit facilement cooptable à d'autres fins :

Not only is PAR [Participatory Action Research] gaining some credence among social scientists, it is also increasingly being promoted or used by international organisations such as the World Bank, government agencies, non-governmental organisations and other groups that constitute civil society. In short PAR appears to be migrating from the margins to the mainstream. While its practitioners may welcome this development, it nevertheless poses some awkward questions. For example, how is the discourse of participation being appropriated and recontextualised by these different organisations? How is participation (and action) being interpreted and implemented? What forms of social organisation are being used to construct the participatory research process? And what kind of knowledge is produced and who benefits from its production? (p. 186)

Si certaines des initiatives de la RP favorisent en effet l'implication et l'émancipation du participant, le danger d'une instrumentalisation de son savoir au profit d'une amélioration de l'efficacité, de la rentabilité et de la compétitivité d'une entreprise, d'une multinationale ou d'un organisme pourrait davantage servir l'accumulation capitaliste que l'empowerment de l'acteur. Écouter la voix des acteurs, n'est-ce pas là en effet le moyen le plus efficace d'accroitre la productivité, la compétitivité ou l'efficacité de son organisation, dans la mesure où la prise en compte de leurs préoccupations permet, au final, de mieux s'adapter à leurs attentes et besoins? Le vocabulaire revêt dès lors une dimension mystificatrice, particulièrement pour les entreprises qui adoptent des processus de RP. Le travail d'équipe, l'attachement à l'entreprise, le leadership distribué et le management " participatif » sont autant de concepts insufflant au travailleur un sentiment à la fois réel et illusoire d'implication et de reconnaissance : réel car il est, en effet, impliqué, consulté et entendu, mais illusoire dans la mesure où l'objectif premier de ces pratiques vise davantage l'efficacité et la rentabilité de l'entreprise que l'émancipation personnelle et collective des travailleurs. Les organismes internationaux influents (Banque Mondiale, Inter-American Development Bank, Asian Development Bank) ne sont pas en reste et cooptent également le langage de la participation et du partenariat (Jordan, 2013, pp.193-195). Les chercheurs universitaires sont ainsi convoqués pour le développement de RP au sein de ces organisations dans le but de favoriser le changement social, la décentralisation des pouvoirs et la prise en compte de la perspective des acteurs sociaux défavorisés : mais simultanément, certains de ces organismes privilégient davantage leurs relations aux grandes entreprises qu'à celles qu'ils entretiennent avec les populations locales, en finançant différents projets qui contredisent leurs propres principes de lutte contre la pauvreté ou d'amélioration des conditions de vie dans les pays en développement 
(Ramachandran, 2019). Cook et Kothari (2001) parlent ainsi d'une «tyrannie de la participation »dans la mesure où, loin de créer l'empowerment et le développement promis, la récupération du langage de la RP par différentes organisations contribue en fait au renforcement des inégalités sociales du fait qu'elle s'attache et se subordonne nécessairement aux objectifs économiques des organismes qui empruntent cette conception.

La question spécifique de la récupération et de la reformulation idéologiques, en contexte capitaliste, de certains concepts ou types de pratiques (dont les visées initiales étaient pourtant progressistes) est particulièrement bien analysée par Boltanski et Chiapello dans Le nouvel esprit du capitalisme (1999). Les auteurs distinguent d'abord deux formes de critique : la critique artiste, liée au mouvement de la contre-culture, selon laquelle le capitalisme contraint la liberté et la créativité des individus, et la critique sociale, souvent d'inspiration marxiste, selon laquelle le capitalisme est vecteur d'injustice. Selon eux, la critique sociale serait tombée en désuétude tandis que la critique artiste aurait prévalu, mais en étant absorbée et récupérée par le management d'une façon qui aurait permis de protéger la légitimité du capitalisme tout en lui donnant de nouveaux instruments d'efficacité (l'engagement et la responsabilité de l'acteur, la valorisation de sa flexibilité et de son adaptation constante au changement, de sa mise en valeur en termes de capital humain). En effet, ce nouvel esprit du capitalisme aurait absorbé la critique «artiste » de façon quasi automatique, $\mathrm{du}$ fait qu'il est lui-même fondé sur des valeurs de liberté et de d'autonomie individuelle : par exemple, l'employé accepte un emploi instable parce que ce type d'emploi lui procure de la flexibilité et de la liberté, il accepte de travailler de longues heures car son travail participe à son épanouissement, etc. Ainsi, son propre exercice de liberté et d'autonomie se confond avec les demandes du nouvel esprit : mais le cadre dans lequel il est permis de s'épanouir est tout de même prédéfini par l'ordre socioéconomique, qui se raffine progressivement pour sans cesse répondre mieux aux critiques, et ainsi assurer le plein engagement du travailleur. Selon Boltanski et Chiapello, ce mouvement de ravalement constituerait le moteur même du capitalisme, mais il ne doit pas être interprété comme une forme de mystification ou d'aliénation de l'acteur :

27 Nous avons voulu rappeler que les hommes font leur histoire, et qu'aucune « cause externe» ne peut s'imposer sans rencontrer dans le tissu social de multiples relais. Aucune globalisation par exemple n'est possible si elle ne prend pas forme dans l'action de multiples personnes qui, travaillant localement à transformer les dispositifs, la font exister peu à peu. (...) La notion d'esprit du capitalisme insiste sur le fait que toute transformation en profondeur du capitalisme, hors période de cataclysmes bien sûr, suppose un accompagnement idéologique qui la rend possible en mobilisant l'enthousiasme de milliers de personnes qui s'y engagent de façon sincère et qui ne sont pas, il faut le souligner, totalement abusées ou manipulées par cette idéologie. Le néocapitalisme a largement répondu à des demandes d'émancipation des hiérarchies et des bureaucraties. C'était le côté attrayant de la transformation. Les gens y ont cru fortement; la transformation a été possible. Tout rabattre sur le néo-libéralisme permet « a contrario » à la critique de jouer une extériorité absolue en se défaussant de toute responsabilité, voire même de toute implication autre que la protestation. (Boltanski et Chiapello, 2000, paragraphes 14-15) 
Cette idée suppose que, si les individus s'engagent dans le déploiement de l'idéologie néolibérale, c'est volontairement, parce qu'ils y trouvent des bénéfices personnels importants. La RP procède d'un mouvement similaire : par sa critique de la domination $\mathrm{du}$ chercheur omniscient, elle intègre les préoccupations des participants et assure ainsi que leurs besoins et attentes soient pris en compte dans toute forme de transformation éventuelle de l'institution dans laquelle ils œuvrent, ce qui permet un raffinement de l'efficacité de l'institution, mais rend peu probable une réelle critique de fond de ses objectifs ou modes de fonctionnement. Et là se joue, précisément, l'enjeu critique de la RP : si l'on admet la capacité d'absorption quasi absolue du potentiel critique des acteurs et de la société civile dans la logique capitaliste, peut-on réellement exclure des tâches d'une sociologie critique le travail de dévoilement et de démystification, de mise au jour de l'aliénation possible et de la domination? Parce qu'il est volontaire et critique, l'acteur est-il pour autant délivré de toute emprise idéologique, de tout rapport de force le désavantageant? Et en contrepartie, une sociologie critique dénonciatrice devient-elle sur-le-champ, comme l'entendent Boltanski et Chiapello dans l'extrait rapporté ci-haut, une "extériorité absolue", irresponsable et uniquement protestataire?

\section{Conclusion}

Nous avons tenté, dans cet article, d'éclairer les impasses critiques de la RP à la lumière de quelques arguments respectifs d'une sociologie critique de la domination et d'une sociologie critique de l'acteur et de la communication : nous avons cherché, d'une certaine manière, à réhabiliter quelques-unes des réflexions de Horkheimer, Adorno et Bourdieu pour comprendre ces impasses qui, selon nous, ne sont pas nécessairement résolues par un appel à Boltanski ou à Habermas. Au final, il nous semble toutefois que, entre une critique du théoricien surplombant et une critique de l'acteur éclairé, une troisième voie soit possible, beaucoup plus nuancée et qui n'ampute ni à l'un, ni à l'autre sa légitimité et sa reconnaissance au sein de la recherche en sciences sociales et des RP. Il s'agit d'une théorie critique conçue comme joute narrative, au sein de laquelle l'objectif n'est plus de témoigner d'une "vérité sociale» qui appartiendrait davantage au chercheur ou à l'acteur, mais plutôt de procéder à une mise en récit de situations sociales capable de faire apparaître de nouvelles significations, de nouvelles ouvertures, de nouveaux possibles. Dans ce mouvement, une description du monde social n'est jamais d'emblée acceptée du simple fait qu'elle provienne de l'expertise du théoricien ou du savoir de l'acteur : elle est plutôt conçue comme une narration capable de susciter l'étonnement et l'indignation, de part et d'autre. Axel Honneth parle en ces termes de la Dialectique de la raison de Horkheimer et Adorno, d'une façon que nous pourrions transposer à n'importe quel récit, qu'il provienne d'un théoricien, d'un chercheur ou d'un acteur :

L'exposition narrative comme la construction de métaphores suggestives comptent parmi les figures rhétoriques qui rendent possibles de tels effets ouvrant de nouveaux ensembles de significations; dans un cas comme dans l'autre, on tente d'ouvrir un nouvel horizon de sens par la concentration sur un ensemble de relations choisies qui font apparaître sous un nouveau jour l'ensemble de nos activités. (...) Par l'effet rhétorique de la condensation et du déplacement, de nouveaux faits doivent devenir visibles dans la réalité sociale. Les destinataires de cette critique ne peuvent s'entendre 
sur la vérité de ces faits qu'après avoir évalué leurs conséquences pour le développement de la société à la lumière de convictions axiologiques concurrentes. (...) Par l'illustration narrative, par le chiasme et par l'art de l'outrance, il s'agit de donner une description de situations de la culture capitaliste devenues familières qui nous les fasse apparaître sous un jour totalement nouveau. L'objectif est de découvrir notre monde comme un contexte de vie sociale dont les orientations et les réalisations peuvent être considérées comme pathologiques puisqu'elles apparaissent, une fois abandonnées nos encombrants préjugés, en complète contradiction avec les conditions de la vie bonne. (Honneth, 2008, pp. 144-149)

Dans cette perspective et au sein des RP qui se donnent spécifiquement un objectif critique de transformation sociale, ni le chercheur, ni l'acteur n'a ainsi le monopole de la vérité sociale et de la "mise en mots " des injustices à décrier ou des situations à transformer : l'accent est posé sur la réception de la critique, en ce qu'elle représente, comme le veut Adorno, une bouteille à la mer qui pourrait n'être jamais trouvée. Dans un jeu mutuel, les vérités du chercheur comme celles de l'acteur pourraient ne jamais être récupérées par l'un ou par l'autre: mais il nous semble que le fait de n'accorder qu'aux chercheurs ou aux acteurs, exclusivement, la possibilité même de lancer ces messages et ces récits contraigne le nouvel horizon de sens que chacun et chacune est en mesure de soumettre à autrui. En d'autres termes, le vrai geste critique de la RP (et, simultanément, de la sociologie critique) n'est peut-être pas celui de combattre le surplomb du chercheur, mais celui de reconnaître à l'acteur sa capacité à lui résister, et la capacité de résistance qu'ils peuvent ensemble former face à un système d'organisation de la recherche qui dicte les règles du jeu et récupère même les plus nobles intentions critiques.

\section{BIBLIOGRAPHIE}

Ancelovici, M. (2009). Esquisse d'une théorie de la contestation. Bourdieu et le modèle du processus politique, Sociologie et Sociétés, v41, n2, pp. 39-61.

Anadón, M. (2007). La recherche participative. Multiples regards, Québec: Presses de l'Université du Québec.

Boltanski, L. (2009). De la critique. Précis de sociologie de l'émancipation, Paris : Gallimard.

Boltanski, L., Chiapello, E. et Laval, C. (2000). Politiques néolibérales et alternatives syndicales. Le nouvel esprit du capitalisme et après? entrevue de Christian Laval avec Luc Boltanski et Eve Chiapello, Institut de recherche de la FSU, décembre 2000. Repéré à : http://institut.fsu.fr/Lenouvel-esprit-du-capitalisme-et.html

Boltanski, L. et Chiapello, E. (1999). Le nouvel esprit du capitalisme, Paris : Gallimard.

Boltanski, L. (1990). Sociologie critique et sociologie de la critique, Politix, v3, n10-11, pp. 124-134.

Bonny, Y. (2014). La recherche-action et la question de l'institution, Forum, n142, pp. 15-24.

Repéré à : https://www.labopraxeo.fr/wp-content/uploads/2014/10/FORUM-142.-Oct-2014.pdf

Questions Vives, $N^{\circ} 33$ | 2020 
Bourassa, M., Bélair, L. et Chevalier, J. (2007). Les outils de la recherche participative, Éducation et Francophonie, v35, n2, pp. 1-11.

Bourdieu, P. (1987). Choses dites, Paris : Éditions de Minuit.

Bourdieu, P. (1984). Questions de sociologie, Paris : Éditions de Minuit.

Bourdieu, P. (1979). La Distinction. Critique sociale du jugement, Paris : Éditions de Minuit.

Bourdieu, P. (1972). Esquisse d'une théorie de la pratique. Précédé de "Trois études d'ethnologie kabyle ", Paris : Droz.

Brydon-Miller, M. (2013). Ethics and action research: Deepening our commitment to principles of social justice and redefining systems of democratic practice. Dans H. Bradbury-Huang \& P.

Reason (dir.), The Sage Handbook of Action Research: Participative Inquiry and Practice (pp. 199-210).

Londres : Sage.

Burns, D. (2007). Systemic action research: A strategy for whole system change, Angleterre : Policy Press.

Cook, B. et Kothari, U. (2001). Participation: the New Tyranny? Londres : Zed Books.

Dardot, P. et Laval, C. (2010). La nouvelle raison du monde. Essai sur la société néolibérale, Paris : La Découverte.

Dewey, J. (1934). Art as experience, Londres : George Allen and Unwin.

Fassin, D. (2009). Une science sociale critique peut-elle être utile? Tracés, n9, pp. 199-211.

Foucault, M. (1990). Qu'est-ce que la critique ? Conférence prononcée à la Société française de philosophie le 27 mai 1978, Bulletin de la Société française de Philosophie, v84, n2, pp. 35-63.

Freire, P. (1991). L'éducation dans la ville, Paris : Paideia.

Frère, B. (2015). Le tournant de la théorie critique, Paris : Desclée de Brouwer.

Giles, H. (2012). Negotiating the boundary between the academy and the community. Dans D. W. Butin \& S. Seider (dir.), The engaged campus: Certificates, minors, and majors as the new community engagement (pp. 49-68). New York: Palgrave Macmillan.

Granosik, M. (2018). Participatory Action Research in Social Work: Towards Critical Reframing, Pensée plurielle, v2, n48, pp. 77-90.

Habermas, J. (1987). Théorie de l'agir communicationnel (Tome I et II), Paris : Fayard.

Habermas, J. (1979). La colonisation du quotidien : sur la situation intellectuelle de l'Allemagne fédérale, Esprit, v12, n36, pp. 34-51.

Hagey, R.S. (1997). The Use and Abuse of Participatory Action Research, Chronic Diseases in Canada, v18, n1, pp. 1-7.

Honneth, A. (2008). La société du mépris, Paris : La Découverte.

Horkheimer, H. et Adorno, T.W. (1947). La dialectique de la raison, Paris : Gallimard.

Horkheimer, M. (1937). Théorie traditionnelle et théorie critique, Paris : Gallimard.

Jordan, S. (2003). Who Stole my Methodology? Co-opting PAR, Globalisation, Societies and Education, v1, n2, pp. 185-200.

Lake, D. et Wendland, J. (2018). Practical, Epistemological, and Ethical Challenges of Participatory Action Research: A Cross-Disciplinary Review of the Literature, Journal of Higher Education Outreach and Engagement, v22, n3, pp. 11-42. 
Lewin, K. (1951). Field Theory in Social Sciences, New York: Harper and Row.

Morrissette, J. (2013). Recherche-action et recherche collaborative : quel rapport aux savoirs et à la production de savoirs? Nouvelles Pratiques Sociales, v25, n2, pp. 35-49.

Mouffe, C. (2000). Deliberative Democracy or Agonistic Pluralism, Social Research, v66, n3, pp. 745-758.

Musil, L. (2018). Social Work and Functions of Participative Action Research, Pensée plurielle, v2, n48, pp. 91-97.

Ramachandran, V. (2019). The World Bank must clean up its act, Nature, 19 mars 2019. Repéré à : https://www.nature.com/articles/d41586-019-00876-6 ?WT.ec_id =NATURE-20190321\&sapoutbound-id =8F151BF7CBC93D51D99A8638EEB0DDCC65F394AF

René, J.-F., Champagne, M. et Mongeau, S. (2013). Allier recherche et participation citoyenne. Enjeux, défis et conditions de réalisation, Nouvelles Pratiques Sociales, v25, n2, pp. 25-34.

René, J.-F., Laurin, I. et Dallaire, N. (2009). Faire émerger le savoir d'expérience de parents pauvres : forces et limites d'une recherche participative, Recherches qualitatives, v28, n3, pp. 40-63.

Rhéaume, J. (1982). La recherche-action : un nouveau mode de savoir ? Sociologie et sociétés, v14, n1. pp. 43-51.

Schön, D. (1983). Le praticien réflexif. À la recherche du savoir caché dans l'agir professionnel, Montréal : Logiques.

Stacey, R. D. (2002). Research perspective: Organizations as complex responsive processes of relating. Journal of Innovative Management, v8, n2, pp. 27-39.

Trom, D. (2008). La crise de la critique sociale vue de Paris et de Francfort, Esprit, n346, pp. 108-126.

\section{RÉSUMÉS}

La nature critique de la recherche participative (RP) est généralement admise d'emblée: en déstabilisant et en reformulant les rapports habituels aux savoirs et les hiérarchies entre experts et acteurs du terrain, la RP contribuerait à fracturer les conceptions traditionnelles du chercheur et de l'acteur/praticien (voire du citoyen) et permettrait le développement du pouvoir d'action du citoyen, la valorisation du savoir du praticien et la prise de conscience des capacités des sujets. Toutefois, et tel est l'objectif du présent texte, les fondements critiques de la RP ne peuvent se soustraire à une réflexion de fond sur ses liens épistémologiques à la notion même de critique en termes sociologiques. Nous analysons ainsi trois «impasses critiques» de la RP (les possibilités d'instrumentalisation des participants, de reconduction des rapports de pouvoir entre chercheurs et acteurs puis de récupération idéologique au sein de ce type de recherche) à l'aide de certains débats qui opposent, en sociologie critique, une sociologie de la domination (Horkheimer, Adorno, Bourdieu) à une sociologie de l'acteur et de la communication (Boltanski, Habermas).

The critical nature of participatory action research (PAR) is generally recognized amongst researchers in human and social sciences: by destabilizing the traditional relationships to knowledge and reformulating the hierarchies between experts and social actors, PAR is seen as an opportunity to develop the social actor's power in society as well as a way to legitimate the actor's knowledge and recognize his critical capacities. However, the critical foundations of PAR must be confronted to a reflection on its epistemological relation, in sociological terms, to the 
very notion of critique. Therefore, we examine in this paper three "critical problems" of PAR (the possibilities of 1-instrumentalizing the participants within PAR, 2- the question of the power relations between researchers and actors and 3- the dangers of an ideological recuperation of PAR's core principles by different organisations) in the light of certain debates in critical sociology that oppose a sociology of domination (Horkheimer, Adorno, Bourdieu) to a sociology of agency and communication (Boltanski, Habermas).

\section{INDEX}

Mots-clés : recherche participative, critique, sociologie critique, domination, acteur social Keywords : participatory action research, critique, critical sociology, domination, agency

\section{AUTEURS}

\section{ARIANNE ROBICHAUD}

professeure, département d'éducation et pédagogie, UQÀM

\section{MARINA SCHWIMMER}

professeure, département d'éducation et pédagogie, UQÀM 